\title{
Aplicación de un Ciclo de Mejora Docente en "Ciencia y Tecnología del Medio ambiente"
}

\author{
Jesús CAMBRollé Silva \\ Departamento de Biología Vegetal y Ecología \\ Universidad de Sevilla \\ cambrolle@us.es \\ ORCID: https://orcid.org/0000-0002-9750-6328 \\ D.O.I.: http://dx.doi.org/10.12795/JDU.2018.i01.71 \\ Pp.: $1272-1289$
}

\section{Resumen:}

El presente trabajo analiza la experiencia de aplicación de un Ciclo de Mejora Docente en la asignatura "Ciencia y Tecnología del Medio Ambiente" (Grado de Ingeniería Agrícola de la Universidad de Sevilla). El ciclo de mejora se llevó a cabo durante la impartición del bloque de contenidos titulado "Interacciones entre poblaciones", que incluye contenidos conceptuales clave para entender en qué medida la integración de conocimientos relacionados con la ecología puede aportar una formación esencial para la adecuada gestión de las explotaciones agropecuarias. La aplicación del modelo metodológico, basado en el trabajo activo en torno al análisis y discusión de casos prácticos, contó con una elevada implicación de los estudiantes. Los resultados resaltan la importancia de convertir la clase en un entorno de trabajo cómodo y ameno para los estudiantes, en el que se sientan libres para expresarse y compartir dudas e inquietudes, como una de las claves para desarrollar exitosamente este tipo de experiencias. 
Palabras claves: Ciencia y Tecnología del Medio Ambiente, Grado en Ingeniería Agrícola, Docencia Universitaria, Experimentación Docente Universitaria, Interacciones Biológicas.

\section{Contexto de la intervención}

La aplicación del Ciclo de Mejora Docente asociado al Curso General de Docencia Universitaria de la Universidad de Sevilla se llevó a cabo durante el primer cuatrimestre del curso académico 2018-2019. La asignatura en la que se aplicó el ciclo, "Ciencia y Tecnología del Medio Ambiente", es obligatoria en el segundo curso del Grado de Ingeniería Agrícola, impartido en la Escuela Técnica Superior de Ingeniería Agronómica de la Universidad de Sevilla. Los objetivos principales de la asignatura se centran en el conocimiento de la estructura y funcionamiento de los ecosistemas naturales y agroganaderos, los efectos de las actividades agrícolas y ganaderas sobre el medio natural, y los métodos de identificación y corrección de los impactos generados por dichas actividades. Como principal problema asociado al bajo índice de aprobados en la asignatura durante los últimos cursos académicos (menos de la tercera parte de los estudiantes matriculados) cabe citar la escasa asistencia a clase del alumnado. La asignatura se estructura en sesiones teóricas (dos clases de una hora a la semana) y prácticas (una clase de dos horas a la semana). El grupo en el que se lleva cabo el Ciclo de Mejora Docente consta de 64 estudiantes matriculados. El Ciclo de Mejora se llevó a cabo durante la impartición del bloque 6 de contenidos, titulado "Interacciones entre poblaciones", en cuatro sesiones desarrolladas durante el mes de octubre. Cabe reseñar que del total de alumnos matriculados, aproximadamente el 30\% no asistió a clase en ninguna de las sesiones teóricas de la asignatura. El diseño del Ciclo de Mejora expuesto en el presente trabajo, tanto en la estructura del diseño, como en la aplicación y evaluación del 
mismo se basa en lo expuesto en el libro "Enseñanza universitaria. Cómo mejorarla” (Porlán, 2017).

\section{Diseño del Ciclo de Mejora Docente}

\section{Mapa de contenidos}

El tema incluye contenidos conceptuales clave para entender en qué medida la integración de conocimientos relacionados con la ecología puede aportar una formación esencial para la adecuada gestión de las explotaciones agropecuarias (uno de los principales objetivos de la asignatura). Así, en una primera fase se desarrollan contenidos conceptuales para entender cómo funcionan las interacciones entre organismos en los ecosistemas naturales, para posteriormente enlazar con una serie de ejemplos sobre interacciones en ecosistemas naturales y en agroecosistemas, incluyendo algunas beneficiosas para las explotaciones agropecuarias; esta última fase sirve como anclaje para mostrar cómo muchas de estas interacciones pueden ser explotadas en beneficio de los agricultores y ganaderos con fines productivos. A partir de dichos ejemplos y del análisis de las diferentes tipologías de interacciones se pretende que los estudiantes desarrollen los contenidos procedimentales detallados en el mapa, tomando conciencia, finalmente, de la necesidad de conservar los ecosistemas naturales y las interacciones que se dan en los mismos, considerándolos como herramientas de valor para la gestión sostenible de los sistemas agropecuarios. El mapa de contenidos queda configurado de manera secuencial en cuanto a los diferentes tipos de contenidos a desarrollar, mostrándose asi una estructura jerárquica en cuanto a las finalidades formativas que se pretenden; fundamentalmente, que los estudiantes aprendan (i) cómo funcionan las interacciones entre organismos 
y (ii) cómo dichas interacciones pueden ser explotadas con fines productivos en los sistemas agropecuarios.

Tras analizarse los diferentes tipos de contenidos, el tiempo de las sesiones y las finalidades formativas del tema a desarrollar, los contenidos se estructuraron en tres bloques: (1) Bases ecológicas de las interacciones intraespecíficas, (2) Bases ecológicas de las interacciones interespecíficas (ambas, en conjunto, responderían a la pregunta clave "¿Cómo funcionan las interacciones entre poblaciones?"), y (3) Interacciones en agroecosistemas y explotación de las mismas en actividades agropecuarias, que respondería a la pregunta clave "¿Cómo pueden explotarse dichas interacciones en los sistemas agropecuarios?".

\section{-Interacciones entre poblaciones-}

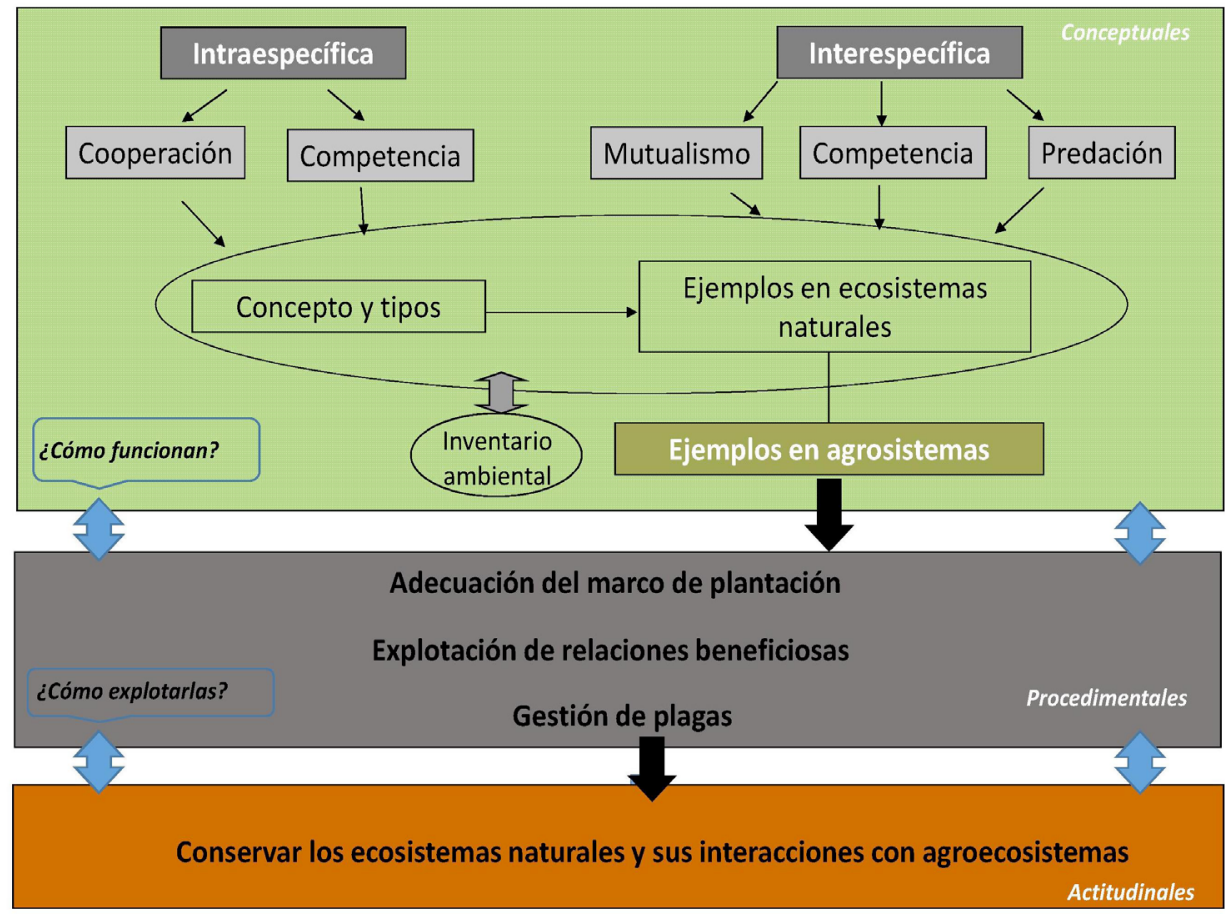

Figura 1. Mapa de contenidos a desarrollar en el Ciclo de Mejora Docente.

Jornadas de Formación e Innovación Docente del Profesorado | № 1 (2018) Esta obra se distribuye con la licencia Creative Commons Reconocimiento-NoComercial-SinObraDerivada Internacional (CC BY-NC-ND 4.0.) 


\section{Modelo metodológico posible y secuencia de actividades}

Se resumen a continuación las fases del modelo metodológico a desarrollar en el Ciclo de Mejora Docente (ver Figura 2):

1.- Presentación breve de noticia o video actual de interés. Se expondrán noticias de interés que guarden una relación con el tema a desarrollar en la sesión. Esta actividad pretende captar la atención del alumnado al inicio de clase y despertar el interés de los estudiantes en el tema a trabajar. 2.- Presentación del caso práctico en el que se basarán las explicaciones de la sesión. Esta fase consiste en la presentación der un caso práctico relacionado con la materia a desarrollar en la sesión, y finaliza lanzando a los estudiantes una pregunta breve y directa sobre el caso. 3.- Análisis y discusión preliminar por los estudiantes del caso práctico. Los alumnos deben analizar el caso y responder a la pregunta formulada tras presentarse el caso práctico, basándose exclusivamente en su propio conocimiento. Se fomenta el análisis crítico de los alumnos y el debate entre ellos, con una mínima intervención del profesor, con el fin de obtener la máxima información posible sobre su estado de conocimiento en la materia. 4.- Explicación de los contenidos teóricos del tema. Se imparten los contenidos conceptuales mediante clase magistral, focalizando las explicaciones en base a la información obtenida en la fase anterior, así como en las posibles preguntas y dudas que los estudiantes formulen. 5.- Análisis conjunto del caso práctico. Se realiza un análisis del caso presentado al inicio de la sesión y analizado preliminarmente por los estudiantes. Dicho análisis se lleva a cabo junto a los estudiantes, fomentando su participación y guiándoles en un análisis global de la materia que conecte sus ideas previas sobre el caso práctico con los contenidos conceptuales impartidos. 


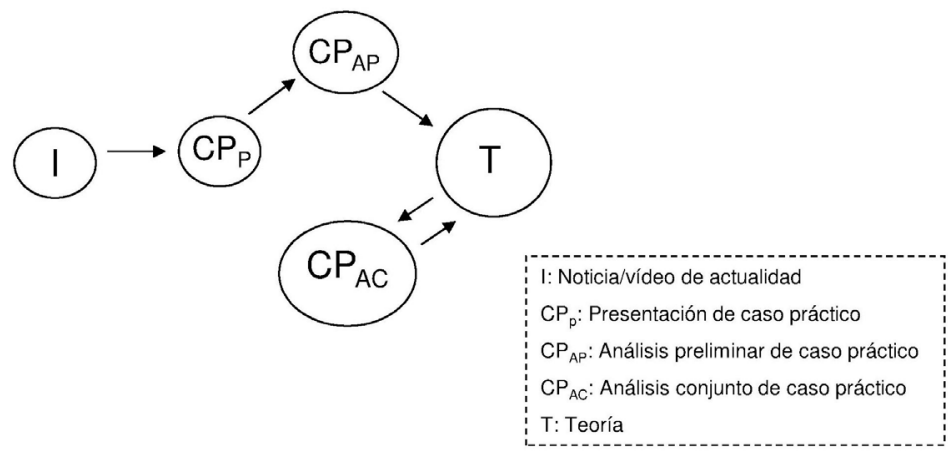

Figura 2. Esquema del modelo metodológico a desarrollar en el Ciclo de Mejora Docente.

En cada una de las sesiones (tres sesiones de 55 minutos y una sesión de 1 hora y 50 minutos) se aplicó el modelo metodológico anteriormente descrito.

Se presenta a continuación la secuencia de actividades detallada, estructuradas en base a las preguntas clave formuladas y a los bloques de contenidos anteriormente detallados:

A.- Pregunta clave: “Cómo funcionan las interacciones entre poblaciones?"

Clase 1 (55 minutos) - Bloque 1: Bases ecológicas de las interacciones intraespecíficas

(1) Realización del cuestionario de ideas previas (10 minutos); (2) Presentación del caso práctico en el que se basarán las explicaciones de la sesión (5 minutos): Se expondrán varios vídeos cortos en los que se visualizan comportamientos animales de cooperación y competencia y se preguntará a los estudiantes sobre la finalidad u objetivo que persiguen tales comportamientos animales; (3) Análisis y discusión preliminar por los estudiantes del caso práctico (10 minutos); (4) Explicación de los contenidos teóricos del tema (15 minutos): Se impartirán 
los contenidos conceptuales sobre los diferentes aspectos relacionados con las interacciones intraespecíficas, profundizando en aquellos aspectos que, en base a la anterior actividad, se detecten como menos claros o ajustados a la realidad en los estudiantes; (5) Análisis conjunto del caso práctico (15 minutos): Se analizarán las respuestas expuestas por los estudiantes en la actividad 3, enlazando sus respuestas con los contenidos conceptuales impartidos y profundizando en nuevos ejemplos de interacciones intraespecíficas.

Clase 2 (55 minutos) - Bloque 2: Bases ecológicas de las interacciones interespecificas

(1) Presentación breve de noticia ó video actual de interés (5 minutos): Dado que la explotación del ganado bovino bravo puede considerarse una forma de interacción, con repercusiones sociales y ambientales, en las sesiones en las que se desarrollará el ciclo se expondrán noticias y vídeos breves relacionados con la tauromaquia, fomentando la conexión de ideas y el debate sobre la temática. En esta sesión, concretamente, se expondrá una noticia breve centrada en la repercusión económica de la actividad; (2) Presentación del caso práctico en el que se basarán las explicaciones de la sesión (5 minutos): Se expondrán varios vídeos cortos en los que se visualizan interacciones entre diferentes especies y se preguntará a los estudiantes sobre las causas que llevan a las diferentes especies a interactuar de las formas mostradas; (3) Análisis y discusión preliminar por los estudiantes del caso práctico (10 minutos); (4) Explicación de los contenidos teóricos del tema (20 minutos): Se impartirán los contenidos conceptuales sobre los diferentes aspectos relacionados con las interacciones interespecíficas; (5) Análisis conjunto del caso práctico (15 minutos): Se analizarán las respuestas expuestas por los estudiantes en la actividad 3, enlazando sus respuestas con los contenidos conceptuales 
impartidos y profundizando en nuevos ejemplos de interacciones interespecíficas.

\section{B.- Pregunta clave: "¿Cómo pueden explotarse dichas interacciones en los sistemas agropecuarios?"}

Clase 3 (1 hora y 50 minutos) - Bloque 3: Interacciones en agroecosistemas y explotación de las mismas en actividades agropecuarias (I)

(1) Presentación breve de noticia o video actual de interés (15 minutos): Continuación de noticias y vídeos relacionados con la tauromaquia, centrado en un debate breve entre defensores y detractores de la actividad; (2) Presentación del caso práctico en el que se basarán las explicaciones de la sesión (15 minutos): Se presentará un inventario extraído de un estudio real de impacto ambiental, y se pedirá a los estudiantes que desarrollen los componentes específicos que deberían describirse en el inventario, y que indiquen, argumentando su respuesta, si consideran necesario incluir en el mismo información sobre las interacciones bióticas; (3) Análisis y discusión preliminar por los estudiantes del caso práctico (30 minutos): En esta fase los alumnos deben analizar el caso y responder a las preguntas formuladas. Se espera que los estudiantes identifiquen los principales componentes específcos que deben incluirse en los inventarios ambientales y que, en base a lo trabajado en sesiones anteriores, identifiquen como prioritaria la información sobre las interacciones bióticas en el medio; (4) Explicación de contenidos teórico-prácticos (30 minutos): Se impartirán los contenidos conceptuales y procedimentales sobre los contenidos del inventario ambiental, profundizando en la importancia de aportar información ecológica sobre las diferentes interacciones bióticas que se dan, y pueden darse, en el medio analizado; (5) Análisis conjunto del caso práctico (20 minutos): Se analizarán las respuestas expuestas por los estudiantes en la actividad 3, enlazando sus respuestas 
con los contenidos impartidos, y profundizando, conjuntamente con los estudiantes, y mediante ejemplos reales, en las repercusiones que puede tener no considerar la información sobre las interacciones bióticas en un estudio de impacto ambiental.

Clase 4 (lunes 5/11/2018) (55 minutos) - Bloque 3: Interacciones en agroecosistemas y explotación de las mismas en actividades agropecuarias (II)

(1) Presentación breve de noticia o video actual de interés (5 minutos): Continuación de noticias y vídeos relacionados con la tauromaquia, en concreto, una noticia centrada en el conflicto entre el colectivo de animalistas y aficionados taurinos; (2) Presentación del caso práctico en el que se basarán las explicaciones de la sesión (5 minutos): Se presentarán varias imágenes en las que se muestran escenas en el ámbito de la agricultura y la ganadería; se pedirá a los alumnos que identifiquen los tipos de interacción que se dan en dichas actividades y que propongan métodos para revertir aquellas interacciones no deseables para los agricultores y ganaderos; (3) Análisis y discusión preliminar por los estudiantes del caso práctico (15 minutos): En esta fase los alumnos deben analizar el caso y responder a la pregunta formulada anteriormente. Se espera que los estudiantes, en base a los conocimientos adquiridos en las sesiones anteriores, se planteen cómo clasificar las diferentes actividades agropecuarias en base a los tipos de interacción que se dan en las mismas, y que asimilen que las interacciones perjudiciales para la producción agricola y ganadera (malas hierbas, predadores y enfermedades) pueden revertirse fomentando otros tipos de interacción (control biológico); (4) Explicación de los contenidos teóricos del tema (15 minutos): Se impartirán los contenidos sobre las interacciones que se dan en el ámbito de las explotaciones agropecuarias. Se profundizará en aquellas interacciones que pueden dar lugar a plagas, 
desarrollando ejemplos reales sobre las consecuencias a nivel de producción de los tratamientos químicos y biológicos; (5) Análisis conjunto del caso práctico (15 minutos): Se analizarán las respuestas expuestas por los estudiantes en la actividad 3, enlazando sus respuestas con los contenidos conceptuales impartidos y profundizando en nuevos ejemplos de interacciones en sistemas agropecuarios, así como las aplicaciones con fines productivistas a las que dichas interacciones pueden dar lugar.

\subsection{Cuestionario de seguimiento de la evolución de los estudiantes}

El cuestionario se basará en las dos preguntas clave a desarrollar en el tema: (i) ¿Cómo funcionan las interacciones entre poblaciones?, y (ii) ¿Cómo pueden explotarse dichas interacciones en los sistemas agropecuarios? Así, el cuestionario integrará tan sólo tres preguntas formuladas con el fin de obtener de los estudiantes la mayor información posible sobre su estado formativo en relación al tema.

1. ¿De qué manera pueden interaccionar los organismos de una misma especie en la naturaleza?

2. ¿De qué manera pueden interaccionar los organismos de diferentes especies en la naturaleza?

3. ¿Crees que alguna de estas formas de interaccionar pueden ser beneficiosas para los agricultores y los ganaderos? ¿Cuáles? Explica cómo. 


\section{Aplicación del Ciclo de Mejora Docente}

\section{Relato de las sesiones}

En general, hubo una buena disposición y una participación considerable en las diferentes actividades planteadas en el Ciclo de Mejora Docente. Las preguntas formuladas tras exponer los casos prácticos relacionados con las relaciones intra e interespecíficas (actividad 2) durante las dos primeras sesiones despertó un claro interés en los estudiantes, dando lugar a un análisis preliminar considerablemente robusto, con una participación elevada. Cabe reseñar que en el grupo había varios alumnos muy activos, lo que hizo que en ocasiones la clase fuera "monopolizada" por las intervenciones de dichos estudiantes. El principal problema durante estas sesiones fue la gestión del tiempo; así, los minutos dedicados "a priori" a las explicaciones de contenidos conceptuales se alargaron, lo cual derivó en que la última parte de los contenidos se impartieron demasiado rápido. En cuanto al análisis conjunto del caso práctico, consecuencia de lo anterior, en ambas sesiones se desarrolló en menos tiempo del pretendido (principalmente en la sesión 1), aunque, en general, se percibió que los estudiantes habían entendido los contenidos trabajados. Por otro lado, la tercera sesión se desarrolló como parte de los contenidos prácticos de la asignatura. Se comenzó proyectando un vídeo corto para introducir un breve debate sobre el tema general escogido como eje principal de la actividad 1; siguiendo la tónica de la sesión 2, hubo una gran participación en el debate posterior al vídeo. Al poder dedicar más tiempo que en las anteriores sesiones a esta actividad, se pudo realizar de manera más ordenada y provechosa, de modo que los estudiantes tuvieron tiempo de desarrollar su postura, argumentarla 
y defenderla frente a la posición de otros compañeros. La fase de análisis preliminar del caso práctico, las explicaciones posteriores y el análisis conjunto del caso también se desarrollaron conforme a lo previsto.

La cuarta sesión, como las dos primeras, se desarrolló en clase de teoría, esta vez con un eje principal centrado en las interacciones en agroecosistemas y la explotación de las mismas en actividades agropecuarias. Cabe resaltar que en la actividad 1, presentación de una noticia de interés y posterior debate, se detectó un aumento de la participación y una mejora en la calidad de las intervenciones; posiblemente ello estuvo relacionado con el hecho de que los estudiantes habían adquirido seguridad a la hora de tratar el tema, seguramente como consecuencia de la experiencia adquirida en sesiones anteriores. Esto me afianzó la idea de que puede resultar positivo desarrollar temas de interés de manera secuencial, en diferentes sesiones, más que ir centrando esta actividad en diferentes temas en cada sesión (como hacía en mi práctica previa). En el análisis preliminar de los estudiantes hubo, además de una alta participación, un número considerable de intervenciones que me indicaron que habían comprendido la materia trabajada en las anteriores sesiones. La explicación de contenidos conceptuales y el análisis del caso práctico se realizaron conforme a lo previsto. Durante la fase final de la sesión se desarrollaron en mayor profundidad los contenidos procedimentales del tema; discutiéndose y analizándose diferentes prácticas agroganaderas de interés que requieren una base sólida de conocimiento sobre los temas impartidos en las sesiones anteriores. 


\section{Evaluación del aprendizaje}

A partir del análisis de los cuestionarios realizados por los estudiantes, las respuestas fueron categorizadas en 3-4 niveles, nombrados con letras, siendo " $A$ " las respuestas correspondientes a un menor nivel de conocimiento, $\mathrm{y}$ " $\mathrm{D}$ " la correspondiente al mayor nivel (ver Tablas 1, 2 y 3). Asimismo, se representaron en forma de escaleras incluyendo los principales obstáculos detectados para alcanzar los diferentes niveles (ver Figuras 3, 4 y 5). Resulta interesante resaltar que las respuestas a la pregunta 2 del cuestionario inicial (centradas en las interacciones interespecíficas) evidenciaron que los estudiantes partían de unas ideas previas considerablemente más confusas que las mostradas en la pregunta 1 (relacionada con interacciones intraespecíficas). Ello parece estar relacionado con la mayor complejidad de la pregunta, pero llama la atención el hecho de que gran parte de los estudiantes habían recibido una formación sobre relaciones interespecíficas en niveles educativos anteriores, evidenciándose que dichos contenidos no habían sido asimilados correctamente. Aunque el análisis de los resultados del cuestionario final muestran que la mayor parte de los estudiantes alcanzaron el nivel más elevado en ambas preguntas, cabe resaltar que en el caso de la pregunta 1 el porcentaje de estudiantes que alcanzó dicho nivel fue superior al 90\%, mientras que en la pregunta 2 el porcentaje fue del $73 \%$, permaneciendo en un nivel intermedio más del 25\% de los estudiantes. En cuanto a la pregunta 3, resulta evidente que corresponde con la materia que mostró mayores dificultades para los estudiantes, de modo que menos del $70 \%$ alanzaron el mayor nivel. 


\title{
Pregunta 1.- ¿De qué manera pueden interaccionar los organismos de una misma especie en la naturaleza?
}

\author{
Tabla 1. Resultados de la evaluación de la pregunta 1 en \\ los cuestionarios inicial y final.
}

\begin{tabular}{|l|c|c|c|c|}
\hline \multirow{2}{*}{ Nivel } & \multicolumn{2}{|c|}{ Cuestionario inicial } & \multicolumn{2}{c|}{ Cuestionario final } \\
\cline { 2 - 5 } & Encuestas & $\%$ & Encuestas & $\%$ \\
\hline $\begin{array}{l}\text { (A) No responde ó solo } \\
\text { hace referencia a inte- } \\
\text { racciones en el ámbito } \\
\text { reproductivo }\end{array}$ & $\begin{array}{c}1,5,12,15, \\
18,23,25,26\end{array}$ & 53,3 & - & 0 \\
\hline $\begin{array}{l}\text { (B) Sólo cita la compe- } \\
\text { tencia, ya sea de manera } \\
\text { explícita indirectamente }\end{array}$ & $4,7,13,19$ & 26,7 & 18 & 6,7 \\
\hline $\begin{array}{l}\text { (C) Sólo cita la coo- } \\
\text { peración, ya sea de } \\
\text { manera explícita o } \\
\text { indirectamente }\end{array}$ & 16,22 & 13,3 & & 0 \\
\hline $\begin{array}{l}\text { D) Cita, directa o indi- } \\
\text { rectamente, tanto la } \\
\text { competencia como la } \\
\text { cooperación }\end{array}$ & 17 & 6,7 & $\begin{array}{c}16,17,19,22,23, \\
25,26\end{array}$ & 93,3 \\
\hline
\end{tabular}

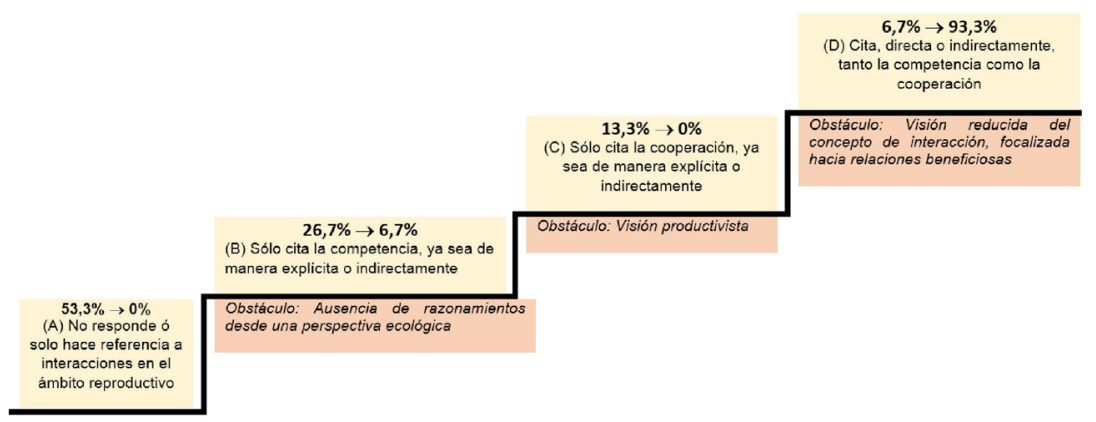

Figura 3. Escalera de evaluación correspondiente a la pregunta 1. Se muestran los porcentajes de estudiantes en cada nivel de aprendizaje en los cuestionarios inicial y final.

Jornadas de Formación e Innovación Docente del Profesorado I № 1(2018)
Esta obra se distribuye con la licencia Creative Commons Reconocimiento-NoComercial-SinObraDerivada $\quad 4.0$ Internacional (CC BY-NC-ND 4.0.) 


\title{
Pregunta 2.- ¿De qué manera pueden interaccionar los organismos de diferentes especies en la naturaleza?
}

\author{
Tabla 2. Resultados de la evaluación de la pregunta 2 en \\ los cuestionarios inicial y final.
}

\begin{tabular}{|c|c|c|c|c|}
\hline \multirow[t]{2}{*}{ Nivel } & \multicolumn{2}{|c|}{$\begin{array}{l}\text { Cuestionario } \\
\text { inicial }\end{array}$} & \multicolumn{2}{|c|}{ Cuestionario final } \\
\hline & Encuestas & $\%$ & Encuestas & $\%$ \\
\hline $\begin{array}{l}\text { (A) Sólo cita la preda- } \\
\text { ción, e indirectamente la } \\
\text { competencia }\end{array}$ & $\begin{array}{c}1,4,12,15 \\
18,19,25,26\end{array}$ & 53,3 & - & 0 \\
\hline $\begin{array}{l}\text { (B) Identifica, aunque de } \\
\text { manera confusa, los princi- } \\
\text { pales tipos de interacción }\end{array}$ & $\begin{array}{c}5,7,13,16 \\
17,22,23\end{array}$ & 46,7 & $4,18,19,25$ & 26,7 \\
\hline $\begin{array}{l}\text { (C) Identifica correcta- } \\
\text { mente los principales tipos } \\
\text { de interacción }\end{array}$ & - & 0 & $\begin{array}{l}1,5,7,12,13,15 \\
16,17,22,23,26\end{array}$ & 73,3 \\
\hline
\end{tabular}

$0 \% \rightarrow 73,3 \%$

(C) Identifica correctamente los principales tipos de interacción

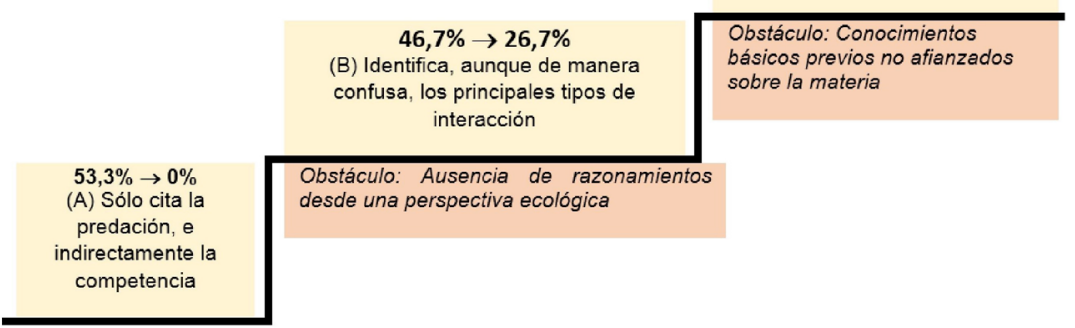

Figura 4. Escalera de evaluación correspondiente a la pregunta 2. Se muestran los porcentajes de estudiantes en cada nivel de aprendizaje en los cuestionarios inicial y final.

Jornadas de Formación e Innovación Docente del Profesorado | № 1 (2018)

(c) Esta obra se distribuye con la licencia Creative Commons Reconocimiento-NoComercial-SinObraDerivada $\quad 4.0$ Internacional (CC BY-NC-ND 4.0.) 


\title{
Pregunta 3.- ¿Crees que alguna de estas formas de in- teraccionar pueden ser beneficiosas para los agricultores y ganaderos?
}

\author{
Tabla 3. Resultados de la evaluación de la pregunta 3
} en los cuestionarios inicial y final.

\begin{tabular}{|c|c|c|c|c|}
\hline \multirow{2}{*}{ Nivel } & \multicolumn{2}{|c|}{ Cuestionario inicial } & \multicolumn{2}{|c|}{ Cuestionario final } \\
\hline & Encuestas & $\%$ & Encuestas & $\%$ \\
\hline $\begin{array}{l}\text { (A) Expone ideas confusas } \\
\text { incluyendo algún ejemplo } \\
\text { concreto sin especificar tipo } \\
\text { de interacción }\end{array}$ & $\begin{array}{c}4,5,17,19 \\
22,25,26\end{array}$ & 46,7 & - & 0 \\
\hline $\begin{array}{l}\text { (B) Identifica, directa o in- } \\
\text { directamente, el control } \\
\text { de plagas como principal } \\
\text { beneficio }\end{array}$ & $\begin{array}{c}1,7,12,13 \\
15,18,23\end{array}$ & 46,7 & $5,7,13,18,22$ & 33,3 \\
\hline $\begin{array}{l}\text { (c) Identifica el control de } \\
\text { plagas como principal bene- } \\
\text { ficio y cita otras relaciones } \\
\text { beneficiosas ligadas a la fase } \\
\text { de plantación y crecimiento } \\
\text { de los cultivos }\end{array}$ & 16 & 6,6 & $\begin{array}{c}1,4,12,15,16 \\
17,19,23,25 \\
26\end{array}$ & 66,7 \\
\hline
\end{tabular}

$6,6 \% \rightarrow 66,7 \%$

(C) Identifica el control de plagas como principal beneficio y cita otras relaciones beneficiosas ligadas a la fase de plantación y crecimiento de los cultivos

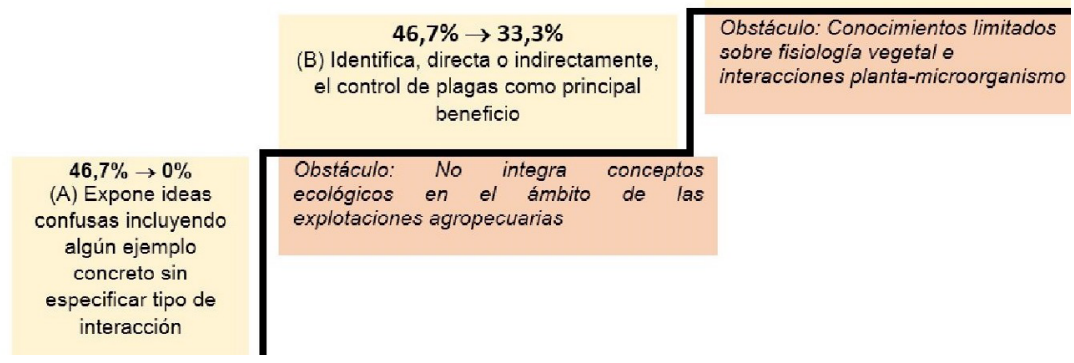

Figura 5. Escalera de evaluación correspondiente a la pregunta 3. Se muestran los porcentajes de estudiantes en cada nivel de aprendizaje en los cuestionarios inicial y final.

Jornadas de Formación e Innovación Docente del Profesorado | № 1 (2018)

Esta obra se distribuye con la licencia Creative Commons Reconocimiento-NoComercial-SinObraDerivada $\quad 4.0$ Internacional (CC BY-NC-ND 4.0.) 


\section{Evaluación del Ciclo de Mejora Docente aplicado}

Tras la aplicación del segundo ciclo de mejora resaltaría que resulta complejo desarrollar el modelo metodológico completo presentado en una clase de una hora, ya que, por mucho que se planifiquen las actividades, puede ser frecuente que las intervenciones de los estudiantes deriven en temas de interés que dilaten el tiempo a dedicar en alguna de las fases. En mi opinión, el escenario ideal es desarrollar este modelo metodológico en una única sesión, principalmente, cuando el tiempo entre sesiones no es corto; sin embargo, en caso necesario se puede adaptar para realizarlo al completo en dos sesiones introduciendo una fase breve al inicio de la segunda sesión en la que se "refresque", en conjunto con los estudiantes, lo trabajado en la sesión anterior. En sesiones de al menos una hora y media creo que es posible, y positivo, desarrollarlo al completo en una única sesión. Por otro lado, en este Ciclo de Mejora se ha desarrollado la primera fase del modelo (presentación de noticia/vídeo de interés y debate breve con los estudiantes) centrando un tema general que se fue analizando de manera secuencial con diferentes vídeos y noticias durante varias sesiones. La sensación general fue muy positiva respecto a modelos anteriores (en los que se trabajaba una noticia o vídeo breve de un tema diferente en cada sesión), ya que los estudiantes parecen ir adquiriendo seguridad, y por tanto pierden el miedo a intervenir más fácilmente, conforme se van viendo inmersos gradualmente en una misma temática. Por ello, como fase introductoria del modelo metodológico desarrollado, se pretende integrar como práctica habitual la presentación secuencial durante varias sesiones de diferentes vídeos y noticias centrados en un tema común desde diferentes perspectivas.

La innovación presentada en el presente trabajo se apoyó en ideas y argumentos expuestos en Bain (2007), de donde derivan los principales principios didácticos que 
han guiado la aplicación de este Ciclo de Mejora Docente, esenciales para desarrollar con éxito experiencias similares, y que pretendo mantener en mi práctica docente habitual. El modelo metodológico desarrollado requiere un alto grado de implicación de los estudiantes en sus diferentes fases; hacerles saber desde un primer momento que su implicación en el aula es tan importante como la del profesor puede resultar clave para la consecución de los objetivos pretendidos. Otra de las claves fundamentales, estrechamente ligada a la anterior, es conseguir que la clase se convierta en un entorno de trabajo cómodo y ameno para los estudiantes, en el que se sientan libres para expresarse y compartir dudas, aprendizajes e inquietudes. A partir de la creación de dicho entorno de trabajo, resulta fundamental fomentar el interés y el desarrollo de una actitud crítica ante problemas actuales y reales relacionados con la materia a impartir. La adecuada elección de los problemas a tratar, si se realiza de manera exitosa, puede aumentar en gran medida el entusiasmo, la motivación y la emoción de los estudiantes en la asignatura, lo que derivará, de acuerdo a los argumentos de Finkel (2008) y Mora (2014), en un mejor rendimiento del proceso de enseñanza-aprendizaje.

\section{Referencias bibliográficas}

Bain, K. (2007). Lo que hacen los mejores profesores universitarios. Valencia, España: Publicacions de la Universitat de València.

Finkel, D. (2008). Dar clase con la boca cerrada. Valencia: Publicacions de la Universitat de València.

Mora, F. (2014). Neuroeducación. Madrid: Alianza Editorial. Porlán, R. (coord.) (2017). Enseñanza universitaria. Cómo mejorarla. Madrid: Ediciones Morata.

Jornadas de Formación e Innovación Docente del Profesorado | № 1 (2018) Esta obra se distribuye con la licencia Creative Commons 